\title{
PROTEINES Adaptation des stratégies d'approvisionnement des filières animales en matières premières riches en protéines
}

\section{Proteins Adaptation of high protein raw material supply strategies for European livestock feeding}

Oléagineux, Corps Gras, Lipides. Volume 9, Numéro 2, 86-91, Mars - Juin 2002, La filière

\begin{abstract}
Auteur(s) : Olivier LAPIERRE, Frédéric PRESSENDA, Cereopa (Centre d'étude et de recherche sur l'économie et l'organisation des productions animales), Ina-PG, 16, rue Claude-Bernard, 75231 Paris Cedex 05, France.
\end{abstract}

Author(s) : Olivier LAPIERRE, Frédéric PRESSENDA

Résumé : L'embargo américain sur le soja en 1973, l'interdiction plus récente de l'utilisation des produits d'origine animale ou la position de la Commission européenne sur l'opportunité d'un « Plan protéine " sont autant d'éléments qui posent la question de l'autonomie protéique des cheptels de I'Union européenne. Le modèle " Prospective aliment ", développé par le Cereopa afin d'analyser les stratégies d'approvisionnement en matières premières des fabricants d'aliments, révèle une augmentation de l'ordre de $40 \%$ de l'utilisation du tourteau de soja en France à l'horizon 2005, par rapport à la période novembre 1999-octobre 2000. Cette évaluation est fondée sur les résultats de modélisation de formules alimentaires et les tendances de production d'aliments composés prolongées jusqu'en 2005. Plusieurs hypothèses ont alors été testées afin d'explorer différentes voies de reconquête de l'autonomie protéique : amélioration de la compétitivité des matières premières face au soja, amélioration de leur qualité nutritionnelle ou encore développement de systèmes d'élevage permettant de réduire la dépendance au soja. Ces réductions ont été estimées pour des voies telles que le remplacement du tourteau de soja par du tourteau de colza dans les rations fabriquées à la ferme dans les élevages laitiers ou porcins (750 $000 \mathrm{t}$ de tourteau de soja économisables). Dans le secteur des aliments composés, nos simulations ont montré l'importance de la thréonine de synthèse pour réduire l'utilisation de soja (350 $000 \mathrm{t}$ de soja économisables), tout comme l'intérêt d'une augmentation du niveau protéique des céréales (250 $000 \mathrm{t}$ de soja économisables pour une hausse d'un point du taux de protéines du blé).

Summary : The US embargo on Soya in 1973, the interdiction of meat and bone meal use or the current position of the European Commission according to the opportunity of a "plant protein" plan bring into question the protein self-sufficiency of European livestock. Compared with the 12 months between November 1999 and October 2000, the model developed by Cereopa for studies on raw products supply of feed compounders indicates an increase of around $40 \%$ of soybean meal use in France for the year 2005. This evaluation is based on modelling and compound feed production trends by the year 2005. Many hypotheses have then been tested to explore different ways of enhancing protein self-sufficiency such as improvement of raw materials competitiveness compared with soya, improvement of their nutritional quality or development of breeding systems allowing soya dependency to be reduced. These reductions have been estimated through such strategies as substitution of rape meal for soybean meal by farmers breeding dairy cattle or pigs $(750,000 \mathrm{t}$ of soybean meal would be saved). Concerning the compound feed sector, our simulations showed the 
importance of synthetic threonine to reduce soya use $(350,000 \mathrm{t}$ of meal), as well as an increase of the protein level of cereals $(250,000 \mathrm{t}$ saved for a one-point protein increase).

Mots-clés : approvisionnement en protéines, nutrition animale, France, Union européenne, consommation de soja, matières premières riches en protéines.

Keywords : protein supply, animal feeding, France, European union, soybean consumption, high protein raw materials.

\section{ARTICLE}

Ébranlées par l'embargo sur le soja de 1973, les filières animales européennes avaient douloureusement pris conscience de la précarité de leur situation en matière d'approvisionnement en protéines. Les évènements récents (interdiction d'utilisation des farines animales et prise de position de la Commission européenne sur l'opportunité d'un "Plan protéine ") ont remis la question de l'autonomie protéique de l'Union européenne sur le devant de la scène. Dans ce contexte, il est utile de procéder au diagnostic de la situation des approvisionnements des filières animales en matières premières riches en protéines et d'évaluer ce vers quoi elle pourrait évoluer. Partant de là, et sur la base d'une connaissance, enfin disponible, du détail des utilisations de tourteaux de soja, il devient possible d'explorer différentes voies de reconquête de l'autonomie protéique et d'en mesurer les conséquences sur les bilans d'approvisionnement.

Évolution récente et perspectives d'évolution

La question de l'approvisionnement en matières premières riches en protéines s'est posée en même temps que les filières animales se développaient en Europe. Il suffit, pour s'en convaincre, de consulter les revues spécialisées des années 1960 où les articles relatifs aux choix des sources d'azote pour l'alimentation des monogastriques (porcs et volailles) étaient nombreux et traduisaient une préoccupation dont les ressorts étaient principalement de nature technique, nutritionnelle en l'occurrence. La question de la dépendance de l'Europe était perçue mais n'apparaissait pas, alors, comme préoccupante. C'est l'embargo sur le soja décrété par les États-Unis en juin 1973 qui joua le rôle de déclencheur de la prise de conscience de la fragilité des stratégies d'approvisionnement de nos filières animales. La crise de 1973, si elle n'a représenté qu'un séisme de relativement courte durée, a surtout permis de révéler le manque d'autonomie dont souffraient chroniquement les filières animales. Elle a incité les responsables politiques de l'époque à promouvoir des moyens de reconquête de cette autonomie. La figure 1 représente un raccourci de l'évolution de la situation française depuis cette période.

La mise en place d'un " Plan protéine ", à la fin des années 1970, fut le principal aboutissement de cette prise de conscience. Fondée sur des mécanismes d'aide à la production et/ou à l'utilisation, cette politique a encouragé le développement des matières premières en rémunérant l'acte de production.

Les bénéfices limités du « Plan protéine » 
Cette politique, même si elle s'inscrivait dans le contexte d'un marché européen où le prix de marché, élevé, des céréales rendait l'accès à l'énergie alimentaire plus onéreux que l'accès à la protéine, a eu un effet significatif sur les utilisations des matières premières produites en Europe. Les protéagineux, les oléagineux métropolitains et la luzerne déshydratée ont été les principaux bénéficiaires de cette politique et ont vu leur niveau de production s'accroître significativement. Peu développées à la fin des années 1970, elles ont atteint, au début des années 1990, un niveau significatif. C'est à cette époque qu'historiquement le taux d'auto-approvisionnement en protéines a été le plus élevé en Europe et en France. Ce remarquable essor de la consommation des produits de l'agriculture européenne a été rendu possible par la mise en place de mesures qui déconnectaient le prix perçu par les producteurs de celui payé par les utilisateurs. Les mécanismes d'aide, le plus souvent indexés sur le prix du tourteau de soja ${ }^{1}$, permettaient à ces produits de maintenir leur compétitivité sur le marché intérieur européen. À la fin des années 1990, le prix perçu par l'agriculteur producteur de pois protéagineux était presque deux fois supérieur à celui effectivement payé, après déduction de l'aide, par le fabricant d'aliments composés pour l'incorporer dans ses mélanges.

Depuis cette période, les contraintes budgétaires de l'Union européenne ont conduit à l'abandon progressif de cette politique de soutien et ont eu pour conséquences de ralentir, voire de contrecarrer, cette dynamique de progrès. Finalement, et compte tenu de l'accroissement de la demande globale en protéines pour l'alimentation animale, la situation d'autonomie protéique n'a que relativement peu progressé depuis 1973. Pour la période 1999/2000, le taux de dépendance de I'Union européenne était encore de près de 70 \%. Pour la France, sur la même période, les matières premières importées représentaient plus de $55 \%$ des protéines consommées, le soja en constituant la quasi-totalité (54\%).

Les risques d'aggravation du déficit protéique européen

Ainsi, les pratiques de formulation des mélanges alimentaires des acteurs des filières animales, même en jouant au maximum sur la substituabilité entre matières premières, ne les ont pas conduits à se détourner massivement du tourteau de soja. Sa composition et son prix de marché expliquent largement cette situation.

La crise récente de l'encéphalopathie spongiforme bovine et ses conséquences en matière d'interdiction d'utilisation des co-produits d'origine animale en alimentation ont mis l'accent sur l'avantage incontestable que ses caractéristiques nutritionnelles confèrent au tourteau de soja. En remplacement de produits à haute teneur en protéines, il s'avère être la seule matière première à pouvoir efficacement compenser l'arrêt de l'utilisation des concentrés protéiques carnés (farines de viande). Les quelques 400000 tonnes de farines de viande utilisées annuellement en France (2 millions de tonnes pour l'Union européenne) ne pouvant guère être substituées que par des quantités encore plus importantes de tourteau de soja.

Cette situation de dépendance continue encore de s'accentuer quand on envisage les évolutions possibles des conditions d'approvisionnement (figure 2). Une estimation réalisée par le Cereopa sur le secteur des aliments composés d'origine industrielle à l'horizon de l'année $2005^{2}$, situe aux alentours de 40 \% l'accroissement de la demande en tourteau de soja par rapport à son niveau durant la période novembre 1999-octobre 2000 (période durant laquelle les farines animales étaient encore autorisées pour l'alimentation des monogastriques). 
C'est donc à une perspective peu favorable à l'amélioration de l'autonomie protéique que se trouvent confrontés l'ensemble des acteurs concernés par l'évolution des stratégies d'alimentation des cheptels en Europe. En dépit du manque d'intérêt que la politique européenne semble aujourd'hui accorder à ce qui reste un élément de fragilisation des productions animales, il reste opportun de s'interroger sur les possibilités de réduction de notre dépendance. Il est nécessaire de repérer les leviers de cette amélioration et d'évaluer les impacts que leur mise en action pourrait induire sur les approvisionnements en matières premières des filières animales.

Exploration de quelques voies de réduction du recours au soja

La recherche de voies efficaces de limitation du recours au soja nécessite de comprendre la nature des mécanismes généraux qui justifient l'utilisation de telle ou telle matière première. La figure 3 présente les principaux facteurs (systèmes d'élevage, qualité et compétitivité des matières premières, limites d'incorporation) qui, en présidant à la formulation des mélanges alimentaires, sont des déterminants majeurs des stratégies d'approvisionnement en matières premières.

Ils nécessitent aussi de connaître avec précision la situation actuelle des utilisations de tourteaux de soja. Des travaux récents du Service central des enquêtes et études statistiques (SCEES) ont permis d'obtenir un éclairage nouveau sur cette question. À partir d'une enquête réalisée en 1996 dans les élevages et sur la base d'extrapolations diverses, il est possible de disposer d'une estimation fiable de la répartition de la consommation de tourteaux de soja entre les différentes spéculations animales d'une part, et entre les différents circuits, aliments composés et utilisation en l'état, d'autre part (tableau 1).

Cette information originale permet de remarquer que le tourteau de soja est valorisé sur un marché assez segmenté. Sans totalement surprendre, ces résultats confirment l'importance des segments " volailles de chair » et " vaches laitières », le premier n'étant quasiment approvisionné que par le biais des fabricants d'aliments composés, alors que le second recouvre aussi d'importantes utilisations en direct par les éleveurs.

C'est en s'appuyant sur cette estimation d'une part, et sur les hypothèses d'évolution de l'activité d'élevage et de la demande en matières premières d'autre part, qu'il est possible de proposer, selon la même clef de répartition, une évaluation du niveau et de la consommation du tourteau de soja envisagée pour 2005 (tableau 2).

Par comparaison avec la situation de l'année 1997, cette estimation confirme l'hypothèse d'une tendance à l'accroissement très sensible de la consommation de tourteaux de soja. Cette augmentation est surtout imputable aux productions de porcs et de volailles de chair et semble grandement le fait du secteur industriel.

C'est sur ces différents segments et de façon éventuellement spécifique pour chacun d'eux que l'on doit étudier les moyens d'une reconquête de l'autonomie protéique. Même si cet exercice spéculatif doit être jugé comme périlleux, il a au moins l'avantage d'ouvrir le champ des possibles et de proposer des éléments de hiérarchisation de l'intérêt potentiel des moyens envisagés. Le tableau 3 présente quelques-uns des résultats ${ }^{3}$ obtenus à l'occasion de l'exploration de différentes pistes correspondant aux trois types de leviers que sont : 
- la compétitivité « prix » des matières premières concurrentes du tourteau de soja ;

- la qualité des matières premières en compétition avec le tourteau de soja ;

- la modification des termes de la demande alimentaire des filières animales.

Les deux premiers correspondent à des mesures à la portée de l'univers des fournisseurs, le dernier, à des mesures relevant d'initiatives des utilisateurs.

Le lecteur sera attentif à ne pas prendre au pied de la lettre les valeurs présentées dans ce tableau. Les mécanismes qui sous-tendent les substitutions entre matières premières sont complexes et leurs conséquences dépendent des contextes dans lesquels on les évalue.

Le levier de la compétitivité " prix »

Les mesures qui permettent de maintenir la compétitivité " prix » des autres sources de protéines sont toujours susceptibles de conforter leur position sur le marché. A contrario, leur abandon, même partiel, réduit considérablement leur chance de s'imposer face au tourteau de soja.

La situation du pois est, à ce titre, remarquable. Utilisé sur le marché français à plus de 2 millions de tonnes au début des années 1990, il l'est, aujourd'hui, à moins de 1,4 million de tonnes. II se trouve pris dans la tenaille inexorable de son manque d'intérêt aux yeux des producteurs, et de sa perte de compétitivité pour les utilisateurs.

Sans conteste, les effets potentiels d'une politique qui voudrait encourager le recours à des matières premières européennes sont loin d'être négligeables.

* Poussé au maximum (ce qui est économiquement irréaliste), l'avantage " prix " qu'une telle politique pourrait leur concéder permettrait de réduire de $45 \%$ la demande en tourteau de soja des fabricants d'aliments composés.

* Appliquée pour rechercher un prix plus compétitif pour la thréonine de synthèse - acide aminé produit industriellement (encadré), ce qui permettrait la généralisation de son incorporation, cette politique permettrait de réduire de 350000 tonnes la consommation de tourteau de soja par les fabricants d'aliments composés.

* Dans les élevages laitiers et porcins utilisateurs de tourteau de soja acheté en l'état, et sans devoir être poussée au-delà du raisonnable, une telle politique pourrait aboutir à des rapports de prix entre matières premières justifiant d'un abandon total de son utilisation.

\section{Encadré}

La thréonine : un acide aminé efficace pour réduire les utilisations de soja

Les possibilités de réduction des incorporations de tourteau de soja dans les aliments composés apparaissent habituellement limitées. Même en favorisant au maximum le recours aux matières premières protéiques d'origine métropolitaine, il reste difficile de se passer totalement de soja. Ses qualités nutritionnelles (densité et équilibre entre nutriments) le rendent partiellement irremplaçable. Cependant, le fait de pouvoir disposer de thréonine de synthèse dans des 
conditions de prix qui assurent sa compétitivité permet d'abaisser encore la limite minimum d'utilisation du tourteau de soja. À l'évidence, sa disponibilité apparaîtrait comme un atout pour des stratégies d'approvisionnement qui chercheraient à minimiser les utilisations de tourteau de soja.

Malgré son efficacité incontestable, le levier « prix » présente des limites qui ne sont pas uniquement budgétaires.

* La capacité de résistance du soja à des baisses de prix de ses concurrents est importante.

* Les avantages " prix » des matières premières produites en Europe ne seront valorisés que s'ils s'accompagnent d'une rationalisation des circuits de mise sur le marché. II convient de créer des conditions telles que, pour l'utilisateur, l'accès à n'importe quel concurrent du soja soit aussi aisé que l'accès au soja lui-même. Ainsi, le faible niveau des utilisations actuelles du tourteau de colza, par les éleveurs laitiers, ne se justifie pas par son rapport de prix au soja, mais pourrait s'expliquer par la difficulté des éleveurs à s'en assurer un approviusionnement régulier.

* Les caractéristiques du tourteau de soja en font une matière première partiellement irremplaçable. C'est principalement sa densité nutritionnelle qui lui confère cet avantage. Avec $48 \%$ de protéines, il est en mesure de satisfaire les besoins d'animaux de plus en plus exigeants (car de plus en plus performants), chose que ses concurrents, moins concentrés en protéines, ne peuvent pas faire.

Le levier " prix " des matières premières concurrentes ne peut pas suffire, à lui seul, à limiter de façon durable l'attrait du tourteau de soja. D'autres voies doivent être envisagées pour fonder une stratégie de reconquête de l'autonomie protéique.

Le levier de la qualité des matières premières

Des mesures visant à améliorer la qualité des matières premières protéiques européennes sont aussi des leviers qui peuvent s'avérer efficaces. Que ce soit par des voies technologiques ou génétiques, elles doivent permettre aux utilisateurs de disposer de matières premières à densité nutritionnelle accrue. Pour ces matières premières, il ne s'agit cependant pas de viser seulement l'amélioration de la qualité protéique. L'énergie reste un constituant dont la valorisation par le marché justifie qu'on y accorde de l'intérêt.

Même si elles ont déjà été largement explorées, mais pas toujours exploitées, les voies d'amélioration de la qualité des tourteaux d'oléagineux métropolitains restent susceptibles d'en améliorer la compétitivité face au tourteau de soja.

* Parmi elles, le tannage, dont la pratique s'est bien développée chez les fabricants d'aliments composés, en dépit du surcoût qu'elle occasionne, est une technologie qui améliore très sensiblement la valeur protéique du tourteau de colza. On peut estimer que sa généralisation est à l'origine d'une importante économie de tourteau de soja (estimée à 250000 tonnes en France en 2000 pour le secteur des aliments composés).

* Les voies d'amélioration qui sont plus ciblées sur l'augmentation de la valeur énergétique des 
tourteaux n'ont pas connu, jusqu'à maintenant, de réel développement. Qu'il s'agisse des techniques de dépelliculage et de décorticage, ou des pratiques qui conduiraient à la production de tourteaux plus riches en huile, elles ont toujours paru peu rentables aux huiliers. Il est possible que, dans des filières de valorisation non alimentaire où les tourteaux trouveraient un débouché sur place, les rapports de prix entre tourteau et huile deviennent plus favorables à la mise en œuvre de ces technologies. Les développements que connaît aujourd'hui cette culture dans l'est de la France pourraient bien être le signe de l'adoption de cette stratégie par des acteurs engagés sur d'autres voies que celle de sa valorisation sur les marchés de l'huile alimentaire.

Dans un autre registre, des démarches qualité qui convaincraient les utilisateurs de repousser les limites d'incorporation qu'ils s'imposent, a priori, sur certaines matières premières (protéagineux, oléagineux métropolitains) se répercuteraient directement sur leurs niveaux moyens d'utilisation et, par voie de conséquence, se traduiraient par une réduction significative des utilisations de tourteau de soja. Cette possibilité concerne autant le secteur des industries de l'alimentation animale que celui des élevages fabriquant eux-mêmes leurs mélanges alimentaires. Rapportée aux éleveurs de porcs fabriquant leur aliment, une telle évolution pourrait aboutir à une substitution totale du tourteau de soja représentant une économie de l'ordre de 340000 tonnes.

Le levier de la qualité des matières premières ne doit cependant pas s'envisager uniquement pour les seules matières premières riches en protéines. Dans des formules d'aliments composés où les céréales occupent, depuis la réforme de la PAC de 1992, une part de plus en plus importante, l'accroissement d'un point en moyenne de leur teneur en protéines ${ }^{4}$ permettrait une économie de tourteau de soja d'environ 250000 tonnes.

Ce levier de la qualité reste un moyen très important d'améliorer l'autonomie protéique de l'Union européenne. II impose cependant d'échapper à une logique de court terme pour envisager des efforts dont les effets ne sont pas toujours spectaculaires mais qui peuvent s'avérer payants sur le long terme.

Le levier de la révision des systèmes de production

Les moyens de limiter les utilisations de soja ne résident pas uniquement dans la capacité de résistance (par le prix ou la qualité) des autres matières premières. Des réorientations partielles des systèmes et règles de production animale sont aussi susceptibles d'induire des effets similaires. Certaines d'entre elles sont déjà d'ailleurs partiellement à l'œuvre.

C'est souvent le niveau recherché de concentration nutritionnelle des mélanges alimentaires qui rend le tourteau de soja si difficile à substituer. Cette concentration est la résultante directe des niveaux de performance des animaux d'élevage que les éleveurs cherchent à atteindre. Avec des exigences moindres, on peut rendre plus facile la substitution du soja par d'autres matières premières.

* C'est ainsi par exemple qu'en comparaison avec les poulets standard, les poulets intermédiaires (animaux à croissance plus lente, abattus à un âge plus élevé et produits dans des conditions de moindre densité) sont susceptibles de valoriser des formules où la présence du tourteau de soja s'avère moins indispensable et où le recours à d'autres matières premières riches en protéines, le pois en particulier, est facilité. À titre d'illustration, la généralisation de ce type de productions aux 
dépens du poulet standard aboutirait à une diminution de la consommation de tourteau de soja d'environ 250000 tonnes.

* Dans la même optique, les choix des niveaux de production par vache visés par les éleveurs laitiers ont aussi des conséquences sur les quantités de soja qu'ils consomment. Là, en revanche, les conclusions sont moins évidentes puisque, ramenée à la production de lait et en comparaison avec ce qu'elle est pour une vache à 7000 litres, la consommation de soja est susceptible de baisser aussi bien sous l'effet d'une diminution du potentiel laitier que sous celui de son augmentation.

Dans ce même champ de la révision des systèmes de production, on doit aussi évoquer la possibilité de remise en cause des règles imposant des teneurs limites en certaines matières premières : les céréales, par exemple, dans la production de volailles label. Ces contraintes, justifiées par le souhait de répondre aux attentes des consommateurs, rendent plus aiguë la question du rééquilibrage protéique des régimes. De ce fait, elles imposent de recourir au tourteau de soja.

De nombreux autres aspects de la production animale sont susceptibles d'influencer la demande en tourteau de soja. L'application de règles de formulation visant à limiter le niveau des rejets en azote et phosphore par les animaux, l'aménagement des capacités de stockage des éleveurs fabriquant leur aliment, le recours à des sources plus diversifiées de matières premières (triticale, farine de luzerne, lupin, etc.) sont autant d'adaptations qui, selon les contextes dans lesquels on les appliquerait, constitueraient des moyens efficaces de reconquête de l'autonomie protéique.

\section{CONCLUSION}

Les filières animales européennes restent encore de grandes consommatrices de soja. Leur efficacité économique n'en pâtit pas quand des conditions ordinaires d'approvisionnement prévalent. Cette situation, en revanche, les rend sensibles à des situations de crise et ne correspond pas totalement aux attentes des consommateurs attirés par le recours à des matières premières locales.

Des possibilités de réduction de cette dépendance à l'égard du soja sont accessibles aux filières animales européennes et françaises. Elles passent par des chemins variés. Attention à l'illusion du " one best way ". À chaque segment d'utilisation peut correspondre une stratégie spécifique de substitution. Elles peuvent imposer des efforts d'amélioration de la qualité des matières premières. Elles réclameront de sortir de la logique du sous-produit et d'admettre que les filières animales sont susceptibles de valoriser la qualité. Elles peuvent conduire à explorer d'autres modes de production dont il faudra démontrer le bien-fondé technique et économique, au-delà de la validation scientifique.

Toutes ces adaptations ne pourront certainement pas être envisagées sur le seul critère de jugement de la consommation de tourteaux de soja. D'autres dimensions du problème participeront de cette décision. Des arbitrages seront à faire où devront être pris en compte les points de vue de différentes catégories d'acteurs : une pratique de recherche du consensus où le génie français n'a pas que des mérites. 
Imagination technique et volonté politique sont des conditions nécessaires au développement de ces changements.

* Cet article présente quelques-unes des conclusions d'une étude réalisée à la demande du ministère de I'Agriculture (DPEI - étude MAP 01.G3.02.01) entre juillet 2001 et mars 2002 et intitulée : " Stratégies d'approvisionnement en protéines des fabricants d'aliments composés. " II s'inscrit dans le prolongement d'une première présentation de ces conclusions qui avait été faite dans le cadre des Rencontres annuelles du Cetiom en novembre 2001.

Notes :

${ }^{1}$ Dans les années 1980, les travaux du Cereopa dans le domaine de l'évaluation de la compétitivité des matières premières avaient été à la base du calcul du panier des matières premières prises en compte pour le calcul de l'aide accordée à la production de luzerne déshydratée.

${ }^{2}$ Cette estimation est fondée :

- sur une évaluation de l'évolution de la demande en aliments composés déduite des tendances observées sur la période 1989-2000 ;

- sur des hypothèses de prix relatifs des matières premières déduites de la confrontation de plusieurs sources (Onic, Commission européenne, etc.) ;

- sur l'utilisation du modèle « Prospective aliment » développé par le Cereopa (http://www.cereopa.com/ presmodelefrance.htm).

${ }^{3}$ L'ensemble du travail réalisé pour le ministère de l'Agriculture en a exploré d'autres.

${ }^{4}$ Accroissement tout à fait envisageable dans l'état actuel.

REFERENCES

index\{references\}

Illustrations 
A

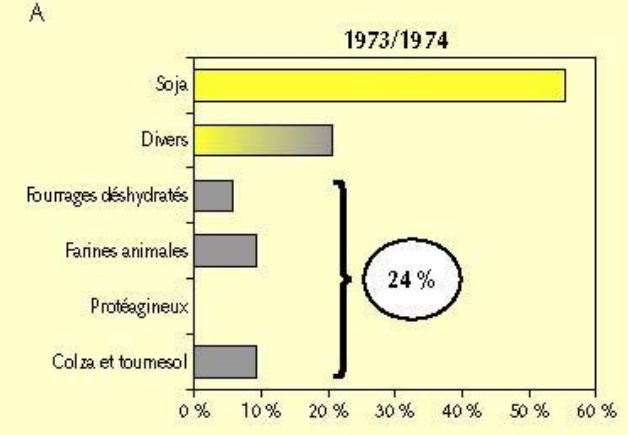

C

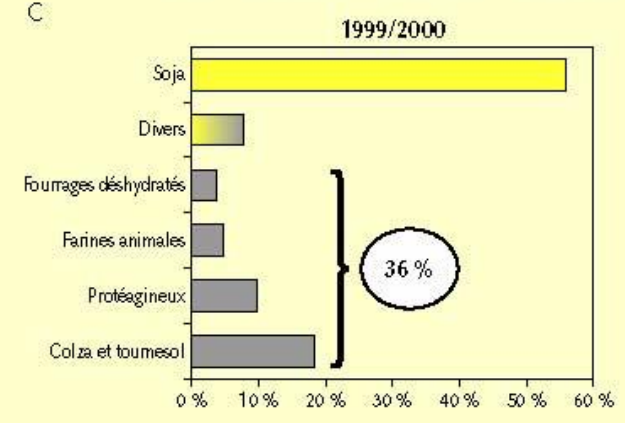

Evolution à long terme des prix du tourteau de soja 48

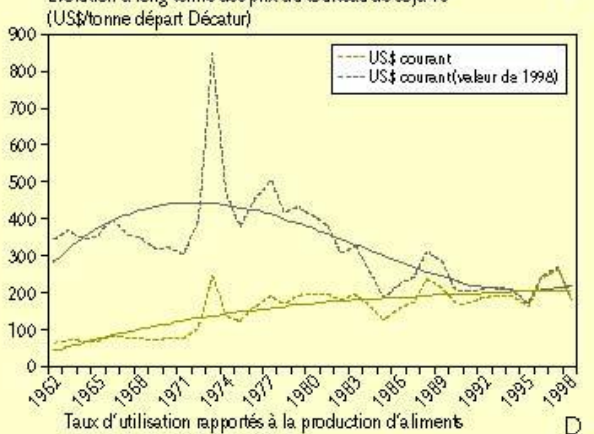

Taux d'utilisation rapportés à la production d'a liment'

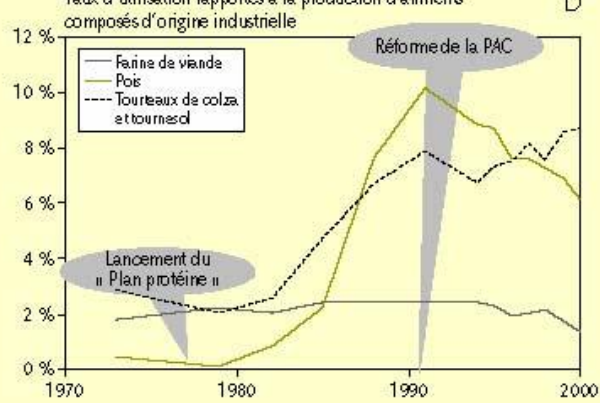

Figure 1. Faits marquants de l'évolution de la situation française en matière d'approvisionnement en matières premières riches en protéines. A : approvisionnement de la France en MRP en 1973/1974 ; B : prix du soja et choc pétrolier de 1973 ; C : approvisionnement de la France en MRP en 1999/2000 ; D : développement des matières premières protéiques européennes.

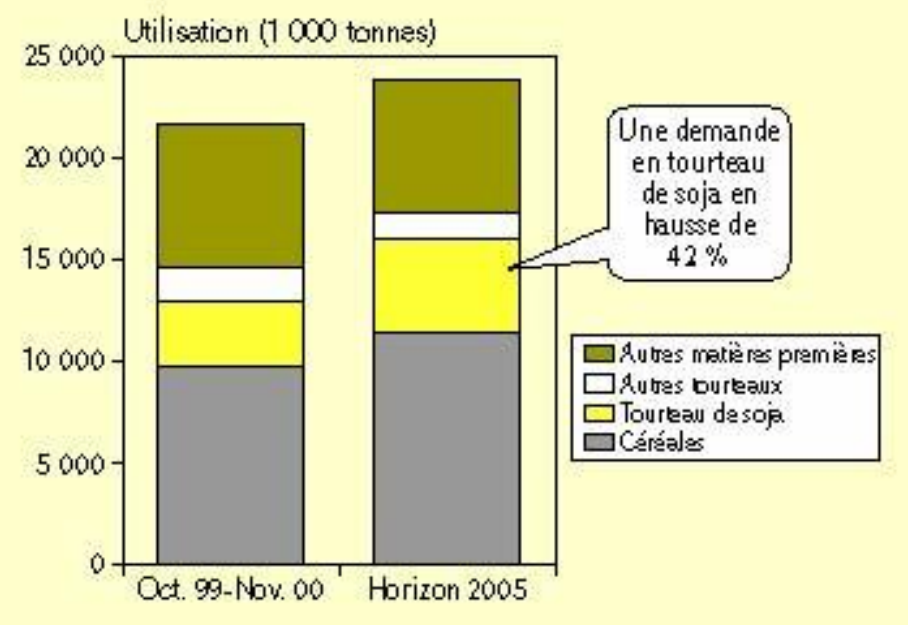

Figure 2. Perspectives d'évolution de la consommation de tourteau de soja par le secteur des aliments composés en France. 


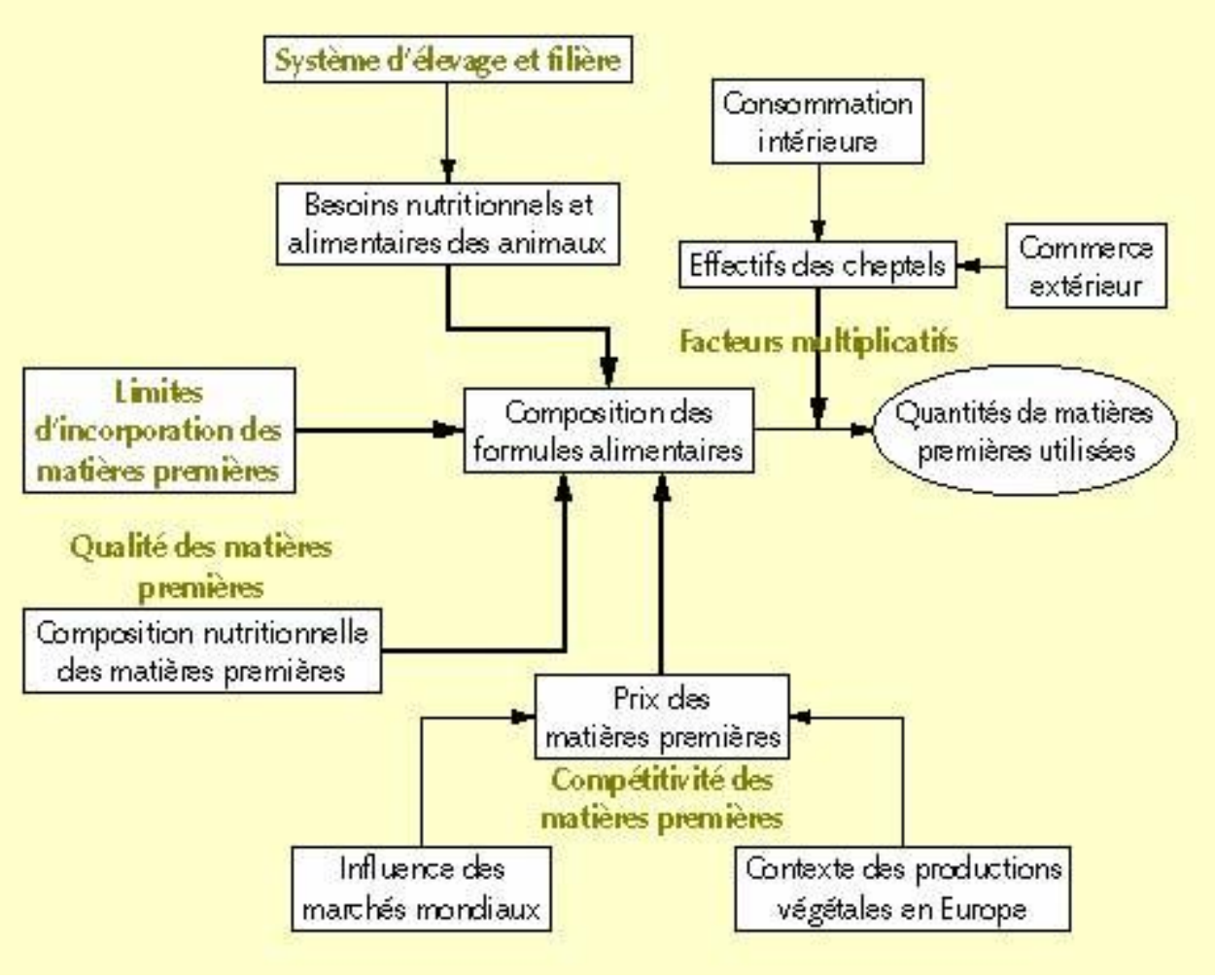

Figure 3. Présentation des déterminants de la composition des formules alimentaires.

Tableau 1. Répartition de la consommation de tourteau de soja en France par type de production.

\begin{tabular}{|l|c|c|c|}
\hline \multicolumn{1}{|c|}{ Type de production } & $\begin{array}{c}\text { Utilisation } \\
\text { industrielle }\end{array}$ & $\begin{array}{c}\text { Utilisation } \\
\text { fermière }\end{array}$ & Total \\
\hline Bovins viande & 240 & 152 & 392 \\
Génisses bit & 56 & 71 & 127 \\
Bovins ait & 610 & 490 & 1100 \\
Porcins & 390 & 179 & 569 \\
Volailles de chair & 1196 & 13 & 1208 \\
Pondeuses & 258 & 60 & 319 \\
Autres & 8 & 46 & 54 \\
Total (1 000 tonn es) & 2758 & 1010 & 3769 \\
\hline
\end{tabular}

Source : SCEES 2001 -enquête FAB et à la ferme du SCEES en 1996 et modèle ALLIX. 
Tableau 2. Estimation de la répartition de la consommation de tourteau de soja en France par type de production à l'horizon 2005.

\begin{tabular}{|l|c|c|c}
\hline \multicolumn{1}{|c|}{ Type de production } & $\begin{array}{c}\text { Utilisation } \\
\text { industrielle* }\end{array}$ & $\begin{array}{c}\text { Utilisation } \\
\text { fermière* }\end{array}$ & Total \\
\hline Bovins viande & 297 & 188 & 485 \\
Génisses bit & 44 & 56 & 100 \\
Bovins bit & 473 & 380 & 853 \\
Porcins & 743 & 341 & 1084 \\
Volailles de chair & 2304 & 25 & 2329 \\
Pondeuses & 556 & 129 & 685 \\
Autres & 17 & 98 & 115 \\
Total (1 000 tonn es) & 4433 & 1217 & 5650 \\
\hline
\end{tabular}

- Résultats du modè le Prospective aliment.

** Par extrapolation sur la base des rapports utilisation E rmière/ utilisation industrielle dL tobkaul.

Tableau 3. Exemples de quelques woies de réduction possibles de la dépendance des filières animales françaises au tourteau de soja.

\begin{tabular}{|c|c|c|}
\hline $\begin{array}{c}\text { Voies possibles de réd uction } \\
\text { du recours au soja }\end{array}$ & $\begin{array}{l}\text { Estimation du tonn age } \\
\text { de soja économisable }\end{array}$ & Secteurs concernés \\
\hline $\begin{array}{l}\text { Utilisation accrue du tourteau } \\
\text { de colza à ba ferme }\end{array}$ & 775000 & $\begin{array}{l}\text { Aliments fabriqués à la ferme } \\
\text { pour vaches bitières et porcins }\end{array}$ \\
\hline Utilisation de b thréonine de synthèse & 350000 & $\begin{array}{l}\text { Aliments com pox́s } \\
\text { pour monogastriques }\end{array}$ \\
\hline $\begin{array}{l}\text { Levée des limites maxima les } \\
\text { d'incorporation en pois et colza }\end{array}$ & 340000 & $\begin{array}{l}\text { Aliments fabriqués à a ferme } \\
\text { pour porcs charcutiers }\end{array}$ \\
\hline $\begin{array}{l}\text { Développement des élevages de poulets } \\
\text { intermédiaires en substitution à l'élevage } \\
\text { de poulets standard }\end{array}$ & 250000 & $\begin{array}{l}\text { Aliments com poés } \\
\text { pour poulets standa id }\end{array}$ \\
\hline $\begin{array}{l}\text { Amélioration de la teneur en protéines } \\
\text { des céréalesd 'un point }\end{array}$ & 250000 & $\begin{array}{l}\text { Aliments com pox́s } \\
\text { pour monogastriques } \\
\text { et rumina nts }\end{array}$ \\
\hline $\begin{array}{l}\text { Augmentation des capacités de stockage } \\
\text { dans lesélevages }\end{array}$ & 170000 & $\begin{array}{l}\text { Aliments fabriqués à b ferme } \\
\text { pour porcins }\end{array}$ \\
\hline Extensification de b production la itière & 90000 & Aliments pour vaches bitières \\
\hline $\begin{array}{l}\text { Développement de la production } \\
\text { de tourteau de colza dépelliculé }\end{array}$ & 80000 & $\begin{array}{l}\text { Aliments com poés } \\
\text { pour porins }\end{array}$ \\
\hline $\begin{array}{l}\text { Éla rgisement descontraintes céréales } \\
\text { dans les formules label }\end{array}$ & 34000 & $\begin{array}{l}\text { Aliments com po és } \\
\text { pour poulet la bel }\end{array}$ \\
\hline Inten sification de b production laitière & 20000 & Aliments pour vaches bitières \\
\hline
\end{tabular}

*Économies de soja estimées (en tonnes) dars l'hypothèse 2005 présentée cirdessus. 